\title{
Development of an integrated digital hand grip dynamometer and norm of hand grip strength
}

\author{
Ho Chang ${ }^{\mathrm{a}, *}$, Chih-Hao Chen ${ }^{\mathrm{b}, \mathrm{c}, *}$, Tung-Sun Huang ${ }^{\mathrm{b}}$ and Chun-Yen Tai ${ }^{\mathrm{a}}$ \\ ${ }^{a}$ Graduate Institute of Manufacturing Technology, National Taipei University of Technology, Taipei \\ 10608, Taiwan \\ ${ }^{\mathrm{b}}$ Department of Thoracic Surgery, Mackay Memorial Hospital, Taipei10449, Taiwan \\ ${ }^{\mathrm{c}}$ Department of Medicine, Mackay Medical College, New Taipei City 25160, Taiwan
}

\begin{abstract}
This study designs and fabricates a novel integrated digital hand grip dynamometer and analyzes collected grip strength data. The dynamometer directly stores collected data in a computer, unlike those on the market that cannot directly store information. A strain gauge load cell is used as a force sensor. The dynamometer is designed to maximize ergonomics. Excitation voltage of the load cell is $5 \mathrm{~V}$, and a $9 \mathrm{~V}$ battery supplies power to its circuit. The signal receiver is National Instruments (NI) data acquisition (DAQ) card that transmits signals to the computer. The operation system is designed using LabView. This study assesses the correlation between variables of collected data. The correlation coefficients for height, weight and palm length were $0.793,0.609$ and 0.715 , respectively, indicating that variables were moderately to strongly correlate with grip strength.
\end{abstract}

Keywords: Hand grip dynamometer, load cell, norm of hand grip strength

\section{Introduction}

Many indices can be used when determining the health of an individual; the most common in Taiwan is the body mass index (BMI). A body's muscle energy system, another standard, is strongly correlated with aging. Aging, a complex physical phenomenon, is related to genetics, physiological structures, lifestyle, hormones and chronic diseases [1]. Regardless of age, physical inactivity results in a decline of muscle bulk and quality, also called muscle degeneration. In the aging process, the function of all organs and tissues gradually declines, eventually reaching death. As such, reliable and economical ways for evaluating aging are essential to the effectiveness of any health care system.

Since muscle energy can be evaluated using physical degeneration-related factors, any method that can examine or test the muscle energy system is a physical degeneration index. Hand grip strength is a

\footnotetext{
* Address for correspondence: Ho Chang, Graduate Institute of Manufacturing Technology, National Taipei University of Technology, Taipei 10608, Taiwan. Tel.: +886227712171; Fax:+886227764889; E-mail: f10381@ntut.edu.tw.

Chih-Hao Chen, Department of Thoracic Surgery, Mackay Memorial Hospital, Taipei10449, Taiwan. Tel.: +886225433535; Fax:+886225232448; E-mail: musclenet2003@yahoo.com.tw.
} 
simple method of assessing muscle energy in the upper extremities [2]. Most prospective studies of hands and aging focused on hand grip strength. Grip strength is an important index for evaluating strength, nutritional status, and degree of disability for the aged. Grip strength of healthy men aged $\geq 65$ declines by $3 \%$ on average each year [3-8]. In 2008 Bohannon synthetically analyzed 7 research papers about hand grip strengths of old people aged over 75. Of them, 270 subjects were males, and 469 females, with all of them using JAMAR hand grip dynamometer as measuring tool. The measuring procedure followed the standardized hand grip strength measuring standards indicated by The American Society of Hand Therapists (ASHT). As a result, normal ranges of hand grip strengths of left and right hands at different age groups were obtained [9]. Kodesh used a hand-held dynamometer (HHD) to determine inter- and intra-tester reliability of strength measurements during maximal electrically induced contractions (MEIC) [10]. Adams investigated the reliability of tongue and hand strength and endurance measurements in old adults using the Iowa Oral Performance Instrument (IOPI) [11]. Seko examined the feasibility of using a hand-held dynamometer (HHD to measure hip extensor strength in the sitting position [12]. Xue assessed the degree and heterogeneity of decline in grip strength in the short term (6 months) and the clinical relevance of short-term decline to long-term decline (2.5 years) in grip strength in disabled older women [13]. Chan used handheld dynamometry to measure Hand grip strength (GS) in community-dwelling men and women aged 5585 from the Hunter Community Study (HCS, N = 2088) and the Sydney Memory and Ageing Study (Sydney MAS, N = 541) [14].

Taiwanese studies of grip strength of the elderly enrolled few subjects, set narrow age ranges, and have no common measurement tool and no standard for grip strength. Thus, comparative and integrated analyses of measured data are difficult. Notably, Taiwan lacks a standard for normal grip strength. Studies of grip strength in other countries used JAMAR hand grip dynamometer, and after analysis, established reference hand grip strength values for those aged 75-99. However, this reference grip strength is inapplicable in Taiwan, mainly because foreigners exercise more, and their genetic background and diets differ greatly. Hence, average grip strength of Europeans and Americans is higher than that of Asians. As the reference values for grip strength are standards used in elderly care, Taiwan needs its own reference values to evaluate the degree and speed of physical degeneration.

The existing hand grip dynamometer, such as JAMAR, mainly records the maximum hand grip strength at a single time. This study further converts the measured hand grip strength to be electronic signal by sensor, and then enter the signal to computer to digitalize the data. In this way, based on the statistical data of hand grip strengths, doctors can judge and analyze the physical conditions of patients. These correlation coefficients between hand grip strength and physical signals, such as height, weight and palm length, are that cannot be acquired from the existing hand grip dynamometer. Nevertheless, it is extremely probable that these physical signals have different clinically meaningful interpretations.

This study applies a novel integrated digital grip dynamometer to generate highly reliable, complete records for hand grip strength, and these data are used to analyze the correlation between data and features.

\section{Experimental details}

Figure 1 shows the hardware of the integrated digital hand grip dynamometer. Its main components are the strain gauge load cell, amplifier circuit, and data acquisition device. LabView software is used to write the program interface. The load cell of this dynamometer is an LMD500 (Futek, Inc., USA). 
This load cell is the sensor of grip strength and the strain gauge. The maximum strain load of the load

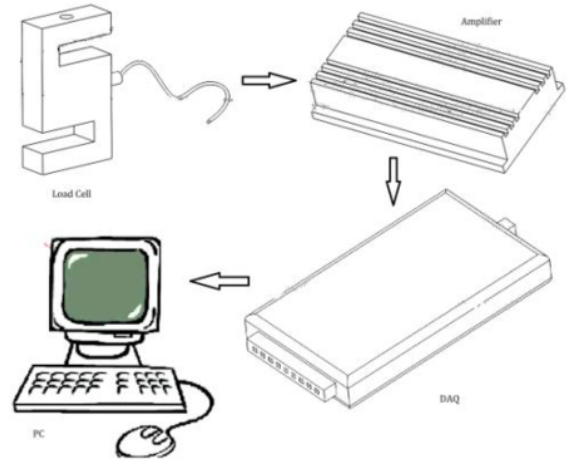

Fig. 1. Hardware structure of the integrated dynamometer.

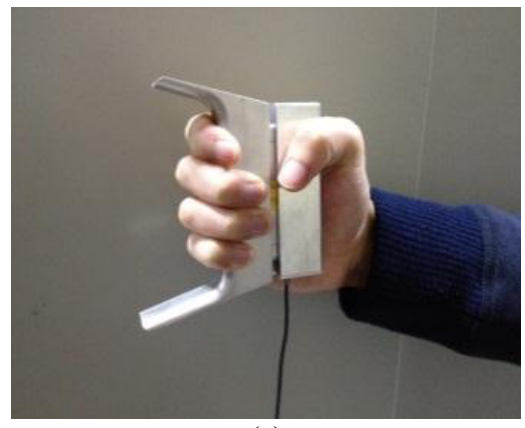

(a)

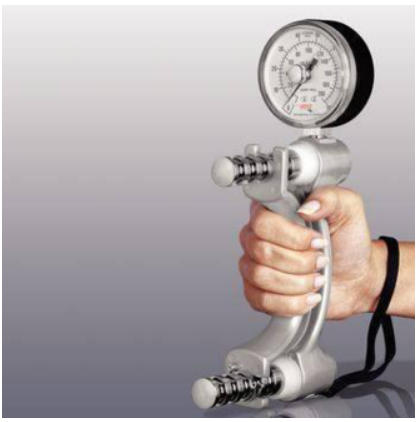

(b)

Fig. 2. Physical images of the (a) integrated digital dynamometer; and (b) the JAMAR dynamometer.

cell is $300 \mathrm{lb}(136 \mathrm{~kg})$. The grip force of most people is less than $100 \mathrm{kgf}$. This designed kit with the length of $5.75 \mathrm{~cm}$ is easy for a hand to hold the hand grip dynamometer. The material of LMD is aluminum alloy. The hand grip dynamometer kit is also made of aluminum alloy, and is divided into upper-part kit and lower-part kit. Figure 2 shows the photograph of the prepared hand grip dynamometer and a commercial dynamometer, JAMAR. The signal receiver is National Instruments (NI) Corporation's data acquisition (DAQ) card, which transmits signal to computer. The operation system was designed by LabView software program to show and store information.

The novel amplifier circuit amplifies signals created by the load cell of the dynamometer. The amplifier circuit uses differential input and differential output composed of 4 operational amplifiers (OPs), and uses a meter amplifier IC (AD620) and amplifier IC (OP07). Two $9 \mathrm{~V}$ batteries supply positive and negative voltages, and a regulator IC supplies stable $5 \mathrm{~V}$ to the load cell as excitation voltage. For data acquisition, a data acquisition (DAQ) card (NI Corporation, USA) is used. Its maximum voltage range is -10 to $10 \mathrm{~V}$; the accuracy of the maximum voltage range of analog input is $138 \mathrm{mV}$; minimum voltage range of analog input is -1 to $1 \mathrm{~V}$; accuracy of the minimum voltage range is $37.5 \mathrm{mv}$; resolution of the analog output is $12 \mathrm{bits}$; and the voltage range of analog output is $0-5 \mathrm{~V}$. Throughout data acquisition, LabView is used as the application interface. This interface comprises the saved route setting, start button, record button, the value of one hand grip strength, and a graph of hand grip strengths. The unit of time for data accumulation is 1 second (sec.). Figure 3(a) shows the amplification circuit, which contains two amplifier ICs and an IC7805 regulator. The top left corner of this figure shows the power input end of the batteries; the bottom left corner shows a 4 PIN joint, 
which is used for excitation voltage for the load cell and the output voltage; and the bottom of the

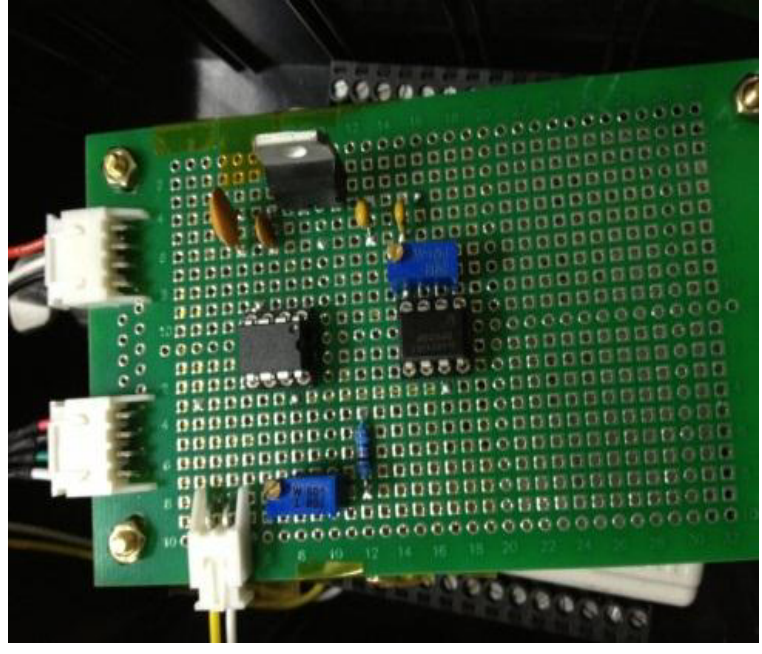

(a)

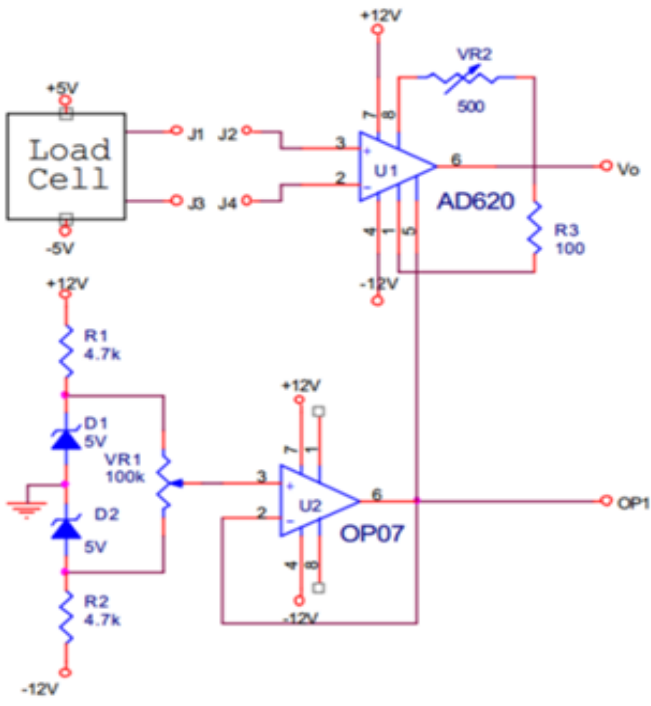

(b)

Fig. 3. (a) The load cell amplification circuit and (b) circuit diagram of the signal amplifier.

figure shows a 2 PIN joint, which transmits amplified signals to DAQ card. Figure 3(b) shows the circuit diagram of the signal amplifier.

As for analytical statistics on hand grip strength data, the study uses Microsoft Excel to establish the original file, which contains the contents of duration and amount of hand grip strength. Meanwhile, Statistical Program for Social Sciences (SPSS12) was used to make descriptive statistics, which shows the various physical data measured from human bodies, as well as the averages, standard deviations, maximum values and minimum values of strengths. In addition, through inferential statistics, we can also know the correlation between hand grip strength and variables of age, height and weight, as well as the difference between them. The significance of statistical data made by this study is requested to be at confidence interval of $99 \%$.

In total, the grip strength of 30 subjects, 23 males and 7 females, was measured using the proposed dynamometer. The age range was 15-30. When assessing grip strength, a subject sat with his/her shoulders slightly contracted. The upper arm hung at $90^{\circ}$ relative to the hand. The wrist was then positioned at $0^{\circ}$ to $-30^{\circ}$ relative to arm, and slightly inclined at $0^{\circ}$ to $-15^{\circ}$ relative to the side of elbow bone (cubitus). The elbow was not supported. During the test, neither visual feedback nor verbal encouragement was given. Their maximum grip strength values were obtained. The age, height, weight and palm lengths were recorded for analysis of correlations with grip strengths.

\section{Results and discussion}

For signal calibration, a push force scale was used to provide the load cell with a weight unit, and voltage was recorded once every $2.5 \mathrm{~kg}$. The change in load and voltage of the load cell was linear. After amplification, tolerance was $\pm 5 \%$.

The nine male testers took the grip strength test over two consecutive days. In demonstrate the practicality and reliability of the proposed dynamometer, these males were asked to repeat the grip 
strength test using the JAMAR dynamometer, and their strength values were compared with those Table 1

Descriptive table of the numerical values of hand grip strengths collected from 30 subjects

\begin{tabular}{lllll}
\hline & Min & Max & Mean & SD \\
\hline Hand grip strength 1 & 17.00 & 63.00 & 39.37 & 11.40 \\
Hand grip strength 2 & 18.00 & 62.00 & 37.77 & 11.17 \\
Hand grip strength 3 & 14.00 & 64.00 & 36.37 & 11.86 \\
Hand grip strength 4 & 18.00 & 56.00 & 35.20 & 10.18 \\
Hand grip strength 5 & 16.00 & 58.00 & 34.33 & 10.18 \\
Height (cm) & 151.00 & 181.00 & 168.32 & 8.36 \\
Weight (kg) & 44.00 & 104.00 & 63.77 & 14.20 \\
Palm length & 14.50 & 20.00 & 17.22 & 1.26 \\
Age & 15.00 & 30.00 & 22.87 & 2.94 \\
\hline
\end{tabular}

obtained with the proposed dynamometer. The difference in grip strength values acquired the two dynamometers was within $2 \%$.

Table 1 shows the descriptive table of the values of hand grip strengths collected from 30 subjects. The table shows the descriptive statistics on 5 times' hand grip strengths, heights, weights palm lengths and ages (including their maximum values, minimum values, averages and standard deviations) of these 30 subjects. As seen from Table 1, under the circumstances of no external stimulation, the average hand grip strengths tend to decrease gradually. For the 5 hand grip strengths recorded for 5 times, the average degree of decrease each time is $4 \%, 3.7 \%, 3.2 \%$ and $2.4 \%$ respectively.

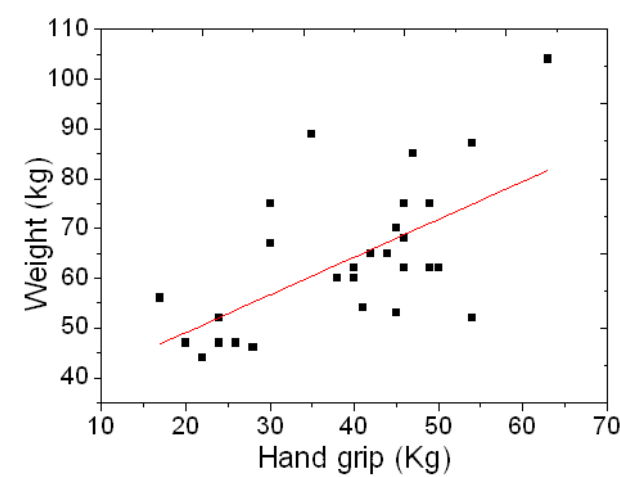

(a)

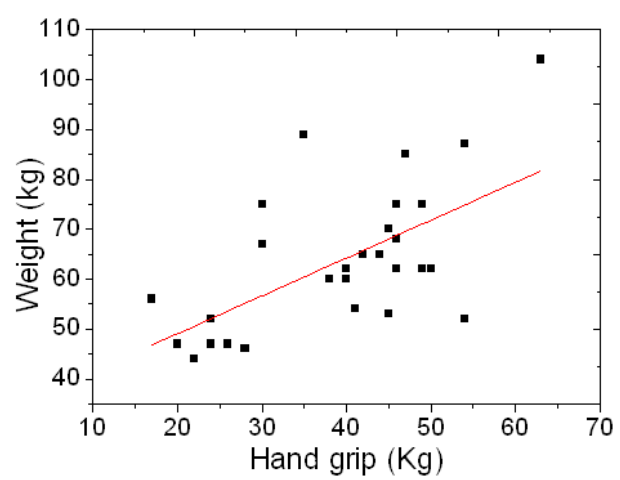

(b)

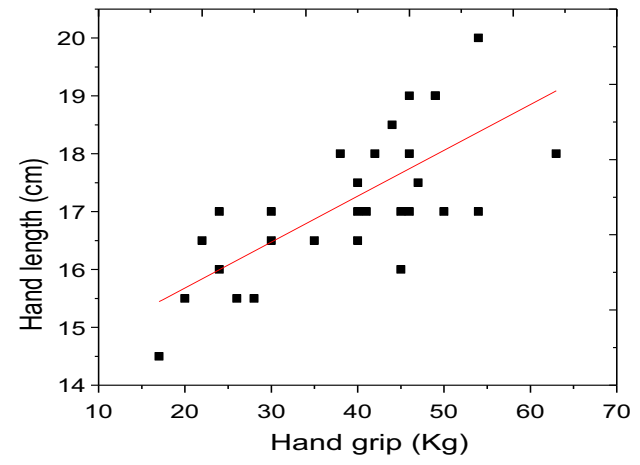

(c)

Fig. 4. Correlation between grip strength and (a) height, (b) weight and (c) palm length. 
Whether grip strength values were correlated with a subject's height, weight or palm length was assessed. If a factor is strongly correlated with grip strength, it has a significant effect on grip strength. Figure 4(a) shows the correlation between grip strength and subjects' height. The coefficient for the correlation between grip strength and height was 0.793 , indicating that the correlation was strong. Figure 4(b) shows the correlation between grip strength and weight. The correlation coefficient of 0.609 , a moderate correlation, demonstrates that the correlation between grip strength and weight is weaker than that between grip strength and height. Figure 4(c) shows the correlation between grip strength and palm length, which, with a correlation coefficient of 0.715 , indicates that the correlation was strong.

Figures 5 show the norm of grip strength data for the left and right grip strengths of 581 Taiwanese males and female subjects. These data were collected from Mackay Memorial Hospital (Taiwan) by the coauthor Dr. Chen, who made measurements using JAMAR dynamometer. Before establishing the norms, the subjects were divided into 17 age groups in the range of 11-95, with each age group covering a 5-year period. Average grip strength for each age group was calculated, and standard deviations were taken as the norms of grip strength for the age group. These data of norms provide Taiwanese with a reference of their health status. Generally, grip strengths of \pm 2.5 times the standard deviation were within the normal range. The grip strength of both males and females decreased as age increased.

\section{Conclusions}

The novel integrated digital dynamometer automatically records grip strength, and can replace the JAMAR dynamometer in clinical studies of grip strength. The difference in reading by the two dynamometers was within $2 \%$. Grip strengths of the 30 subjects aged 15-30 were collected, and their correlations with the height, weight and palm length of subjects were obtained. The correlation between grip strength and height was strong $(0.793)$; the correlation between grip strength and weight was moderate (0.609); and the correlation between grip strength and palm length was strong $(0.715)$.

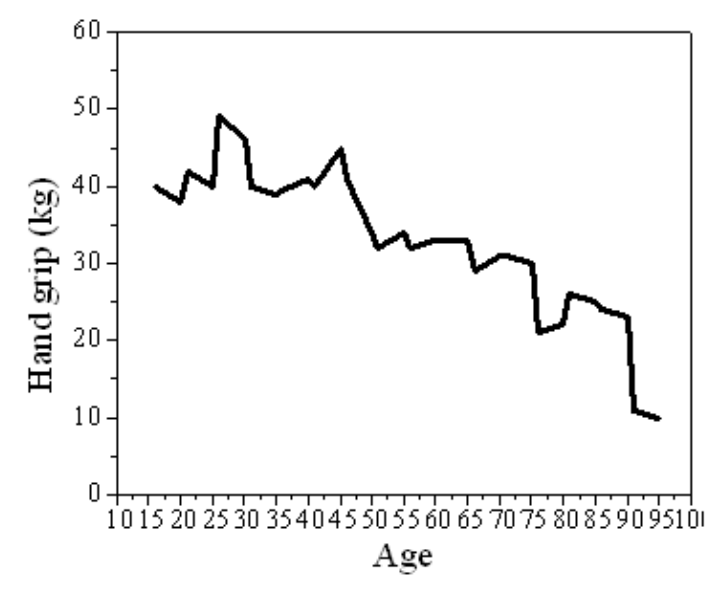

(a)

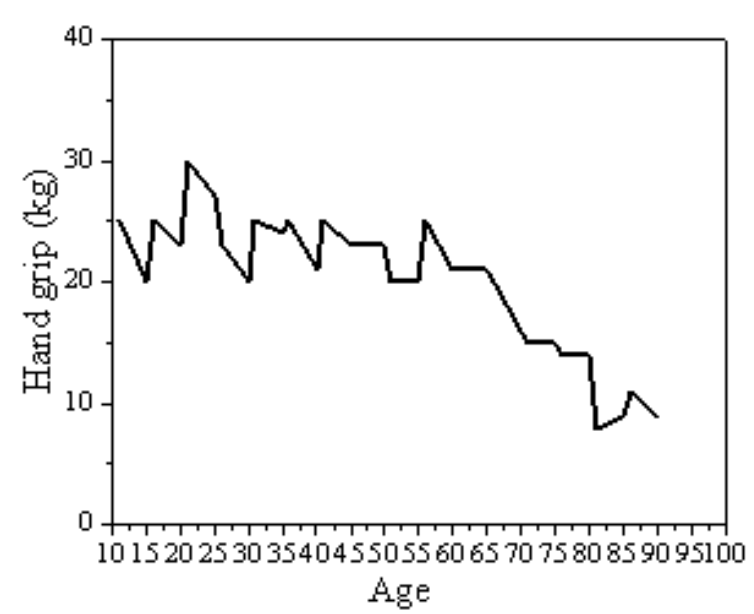

(b)

Fig. 5. Average grip strengths of (a) Taiwanese males and (b) females at different ages. 


\section{Acknowledgment}

The authors would like to thank the National Taipei University of Technology and Mackay Memorial Hospital, Republic of China, Taiwan, for financially supporting this research under Contract No. MMH-TT-10212.

\section{References}

[1] R. Roubenoff, Origins and clinical relevance of sarcopenia, Canadian Journal of Applied Physiology 26 (2001), 78-89.

[2] D.A. Kallman, C.C. Plato and J.D. Tobin, The role of muscle loss in the age-related decline of grip strength: Crosssectional and longitudinal perspectives, The Journal of Gerontol 45 (1990), 82-88.

[3] E.J. Bassey and U.J. Harries, Normal values for handgrip strength in 920 men and women aged over 65 years, and longitudinal changes over 4 years in 620 survivors, Clinical Science 84 (1993), 331-337.

[4] R.J. Shephard, W. Montelpare, M. Plyley, D. McCracken and R.C. Goode, Handgrip dynamometer, cybex measurements and leanmass as markers of the aging of muscle function, British Journal of Sports Medicine 25 (1991), 204-208.

[5] M.G. Bemben, B.H. Massey, D.A. Bemben, J.E. Misner and R.A. Boileau, Isometric muscle force production as a function of age in healthy 20- to 74-year-old men, Medicine and Science in Sports and Exercise 23 (1991), 1302-1309.

[6] A.A. Vandervoort and K.C. Hayes, Plantarflexor muscle function in young and elder woman, European Journal of Applied Physiology and Occupational Physiology 58 (1989), 389-394.

[7] R.W. Bohannon, Reference values for extremity muscle strength obtained by hand held dynamometry from adults aged 20 to 79 years, Archives of Physical Medicine and Rehabilitation 78 (1997), 26-32.

[8] S.K. Hunter, M.W. Thompson and R.D. Adams, Relationships among age-associated strength changes and physical activity level, limb dominance, and muscle group in women, The Gerontological Society of America 55 (2000), B264B273.

[9] R.W. Bohannon, Hand-grip dynamometry predicts future outcomes in aging adults, Journal of Geriatric Physical Therapy 31 (2008), 3-10.

[10] E. Kodesh and Y. Laufer, The reliability of hand-held dynamometry for strength assessment during electrically induced muscle contractions, Physiotherapy Theory and Practice 31 (2015), 61-66.

[11] V. Adams, B. Mathisen, S. Baines, C. Lazarus and R. Callister, Reliability of measurements of tongue and hand strength and endurance using the Iowa Oral Performance Instrument with elderly adults, Disability and Rehabilitation 37 (2015), 389-395.

[12] T. Seko, T. Kumamoto, Y. Takahashi, R. Kaneko, M. Tanaka, M. Shida and T. Ito, The feasibility of a measurement method using a hand-held dynamometer of hip extensor strength in the sitting position, Rigakuryoho Kagaku 30 (2015), 75-79.

[13] Q.L. Xue, J.M. Guralnik, B.A. Beamer, L.P. Fried and P.H. Chaves, Monitoring 6-month trajectory of grip strength improves the prediction of long-term change in grip strength in disabled older women, The Journals of Gerontology, Series A, Biological Sciences and Medical Sciences 70 (2015), 367-373.

[14] J.P.L. Chan, A. Thalamuthu, C. Oldmeadow, N.J. Armstrong, E.G. Holliday, M. McEvoy, J.B. Kwok, A.A. Assareh, R. Peel, S.J. Hancock, S. Reppermund, J. Menant, J.N. Trollor, H. Brodaty, P.R. Schofield, J.R. Attia, P.S. Sachdev, R.J. Scott and K.M. Mather, Genetics of hand grip strength in mid to late life, Age 37 (2015), 1-10. 